

\title{
IDENTIFYING TURBULENT STRUCTURES THROUGH TOPOLOGICAL SEGMENTATION
}

\author{
Peer-Timo Bremer, Andrea Gruber, \\ Janine C. Bennett, Attila Gyulassy, Hemanth Kolla, \\ JACQUEline H. Chen AND RAY W. GROUT
}

\begin{abstract}
A new method of extracting vortical structures from a turbulent flow is proposed whereby topological segmentation of an indicator function scalar field is used to identify the regions of influence of the individual vortices. This addresses a long-standing challenge in vector field topological analysis: indicator functions commonly used produce a scalar field based on the local velocity vector field; reconstructing regions of influence for a particular structure requires selecting a threshold to define vortex extent. In practice, the same threshold is rarely meaningful throughout a given flow. By also considering the topology of the indicator field function, the characteristics of vortex strength and extent can be separated and the ambiguity in the choice of the threshold reduced. The proposed approach is able to identify several types of vortices observed in a jet in cross-flow configuration simultaneously where no single threshold value for a selection of common indicator functions appears able to identify all of these vortex types.
\end{abstract}

\section{Introduction}

Defining and extracting vortices from a turbulent flow is a long standing challenge with implications for a wide variety of applications including study of turbulent boundary layers (e.g., del Álamo et al. [7]) and turbulence-chemistry interaction (e.g., Grout et al. [12]). Several intuitive descriptions of a vortex have been articulated. An early description of a vortex was as a coherent volume of material spinning around a common core [19]. A more recent description is as a coherent structure of the turbulent flow field; a connected, large-scale fluid mass with phasecorrelated vorticity over its spatial extent [15]. Directly extracting vortices based on such definitions has proven practically infeasible especially in the complex, time-dependent flows of greatest interest. Instead, a number of indicator functions have been proposed such as vorticity magnitude [1;9], eigenvalues of the velocity gradient tensor [6], the second invariant of the velocity gradient tensor [14; 18],

MSC2010: primary 76-02, 65D18, 68U05; secondary 76F40, 76F65, 68U10.

Keywords: segmentation, vortex identification, topology, turbulence. 
and the $\lambda_{2}$ criterion [16]. Given any of these derived quantities, vortices are defined using isosurfaces at a particular function threshold. While each indicator function has particular advantages and disadvantages they share two common problems. First, selecting a threshold determines both the strength of the vortices one is interested in as well as their spatial extent. A single threshold is rarely meaningful throughout the entire flow and a given threshold selection can have undesirable consequences. For example, when a weak threshold is selected, high strength vortices can balloon and ultimately merge erroneously. Conversely, when a strong threshold is selected it can cause lower-strength vortices to be ignored. Second, the common indicator functions are known to have a large dynamic range in which case the absolute strength of a vortex may not necessarily be equivalent to its importance. For example, in our test data involving a flame in a turbulent jet in cross flow configuration, the jet break-down region contains significantly stronger vortices in absolute terms than the wake vortices below. However, the wake vortices are an essential part of the description of the overall flow pattern. Combined, these two issues lead to the dilemma of choosing a strong threshold that ignores many important features or a weak one which includes a large number of spurious and/or potentially severely distorted features (see Figures 3 and 4).

In practice, there typically does not exist an optimal threshold, which leads to frequent criticism about the influence of the ultimate choice of threshold on the results of a subsequent analysis [16]. Álamo et al. [7] found this problem particularly frustrating in their analysis of a turbulent boundary layer. In order to compute the volume fraction occupied by vortex regions across a boundary layer, they noted that threshold values of their indicator function suitable for the near wall region detected few vortices in the outer part of the boundary layer. Furthermore, when suitable thresholds for the outer part were selected, the near wall region became cluttered and obfuscated. To mitigate this effect, they scaled the threshold used based on the standard deviation of their indicator function across planes parallel to the wall (i.e., their threshold was dependent on the distance from the wall). This approach was very effective although it depended on leveraging the single direction of inhomogeneity in their specific configuration.

To avoid ambiguities associated with ad hoc vortex extraction methods this paper proposes a general, simple, yet highly flexible vortex detection technique based on the topological analysis of an arbitrary scalar indicator function. A key advantage of our approach is that it decouples the identification of a vortex from the specification of its extent. We first find seeds for all potential vortices as local maxima/minima of the indicator function. Subsequently, we use a topological encoding of the indicator function to optimize a local threshold for each vortex according to some user defined criterion such as size or volume constraints, relative thresholds, or deviations from some assumed model. The resulting vortices can then be explicitly included or 
excluded from any analysis depending on various selection criteria such as size, strength, location, or shape. Our contributions in detail are:

- a new methodology for defining and extracting vortices using topological encodings;

- using a simple but effective metric called relevance to define vortices in terms of a relative threshold that is scaled according to the per-vortex extrema rather than the global indicator range; and

- a case study using a complex, highly turbulent flow that demonstrates the effectiveness of the relevance metric in extracting well-formed, nonoverlapping vortices from many standard indicator functions while requiring no a priori knowledge of the flow geometry.

The remainder of this paper is organized as follows: we first introduce the necessary mathematical concepts and show how a broad range of vortex detection techniques can benefit from topological encodings. We then introduce relevance as an example of a simple to compute metric designed to improve upon traditional thresholding. After a brief survey of common indicator functions, we show how the relevance metric and topological segmentation improve on simple thresholding for a representative direct numerical simulation (DNS) dataset. We conclude with a discussion of the generality of the approach and future work.

\section{Topological segmentations}

This section introduces some basic concepts from scalar topology used throughout the discussion. We then show how vortices defined through any indicator function are equivalent to so called sub- or superlevel sets of the indicator and how these can be efficiently encoded in a merge tree. The advantage of the topological techniques is that they partition the domain into isolated features independent of feature strength. Finally, we define a general class of tree transforms that lead to a notion of localized thresholds and define relevance as a particularly simple yet powerful transform to define vortex extents.

Merge trees. Given a connected domain $\mathbb{M}$ and a smooth function $f: \mathbb{M} \rightarrow \mathbb{R}$ the region $\operatorname{Sup}_{f}(c)=\{p \in \mathbb{M} \mid f(p) \geq c\}$ of $\mathbb{M}$ with function value greater $c$ is called the superlevel set of $c$. We call a connected component of a superlevel set a supercontour. The merge tree of $f$ encodes the evolution of the supercontours as $c$ is swept from $\infty$ to $-\infty$. Each time $c$ passes a local maxima of $f$ a new supercontour is created and supercontours merge at selected saddles sometimes referred to as merge-saddles. Collectively, this structure is typically represented as a tree with local maxima forming the leaves, merge saddles the internal nodes, and the global minimum of $f$ the root (see Figure 1(a)). The arcs of this tree 


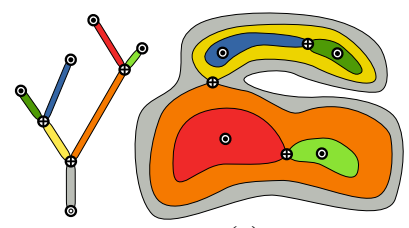

(a)

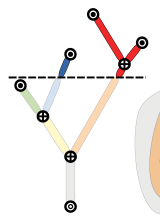

$\odot$

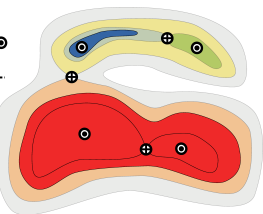

(b)

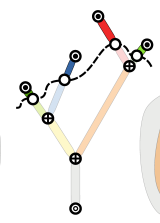

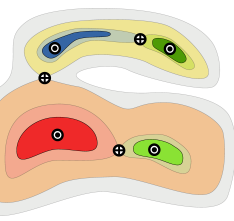

(c)

Figure 1. (a) A merge tree forms a compact encoding of an indicator function. It encodes the merging of contours as the function is lowered through its range. Each branch represents a portion of the domain as indicated by the colors. (b) The selection of a threshold is equivalent to performing a horizontal cut through the tree. Each subtree corresponds to a vortex. (c) Rather than use a single global threshold, localized thresholds can be used to identify the extents of vortices.

represent evolving supercontours that may change their shape but do not touch other supercontours. Equivalently, the sublevel sets, $\operatorname{Sub}_{f}(c)=\{p \in \mathbb{M} \mid f(p) \leq c\}$ give rise to the split-tree containing local minima as leafs, split-saddles as internal nodes, and the global maximum as root.

Given an indicator function $I$ (see Section 3 ) vortices are traditionally defined using a threshold $c$ as regions with the $I \geq c$ or $I \leq c$, or equivalently as the super-/subcontours of $I$. The following discussion will assume the former case, concentrating on superlevel sets but the results for sublevel sets are symmetric.

A merge tree that encodes the indicator function topology forms a highly compact representation of vortices at all thresholds [2]. Given the duality between vortices and supercontours it is convenient to think of choosing a threshold as a cut through the tree. More specifically, consider laying out the tree vertically according to the function values of the nodes. The selection of a threshold is equivalent to drawing a horizontal line through the tree cutting it into a forest of subtrees (Figure 1(b)). Each subtree corresponds to a vortex, and therefore, finding a good threshold is equivalent to finding an appropriate cut through the tree. The challenge of finding a global horizontal cut through the tree nicely illustrates the issues that can arise when choosing a global threshold to define all vortices. Selecting a high cut will miss all lower branches of the tree, yet moving the cut too low will result in overly large (or rather high) subtrees that no longer represent meaningful vortices. Instead, we propose to use the flexibility of merge trees to define local thresholds.

Local thresholds. The main insight from the discussion above is that there is no need to use a horizontal cut to define vortices. In fact any disjunct set of subtrees will define a set of vortices, where each vortex is given by the supercontour that contains the root of its corresponding subtree. Therefore, selecting subtrees is one convenient way of defining local thresholds. Nevertheless, the problem of finding a "good" set of local thresholds remains challenging. 
As mentioned in Section 1, traditionally the threshold performs two important tasks. It defines which regions of the data set are considered "vortex-like" and it determines the spatial extent of a vortex. For global thresholds these two choices are inextricably linked resulting in two competing and sometimes mutually exclusive sets of goals. Local thresholds allow us to ignore the first condition of which regions to consider. Instead, we initially consider all possible vortices as identified by all leaves in the merge tree, i.e., all regions with a local maximum of the indicator. Conceptually, one can consider this a cut that runs just below all leaves of the tree which corresponds to a (maximal) set of tiny, "seed" vortices. Subsequently, we can determine the spatial extent of each of these vortices by lowering the local threshold, which effectively grows and merges vortices. This results in an arbitrary cut of the tree which intersects the path from each leaf to the root exactly once (Figure 1(c)). Since in this manner we are guaranteed to not miss a potential vortex one can use an arbitrary user defined metric to define the "optimal" spatial extent and the corresponding local threshold. As well be demonstrated later, the results tend to be insensitive to the choice of the metric.

Metric indicators. Defining a cut as described above provides a highly flexible means to select vortices. For example, one could use a goodness of fit to an idealized structure, such as an Oseen vortex to identify well formed structures. However, the merge tree also allows to go beyond identifying a single set of vortices. In particular, rather than finding an "optimal" cut point along each path from a leaf to the root one can evaluate a given metric for all possible subtrees. As long as these values respect the structure of the merge tree, i.e., each subtree being assigned a higher metric than its parent, the metric itself effectively defines a new indicator function. Conceptually, assigning values to all subtrees defines not just a single cut through the tree but a nested set of cuts according to the metric. One can now "straighten" these cuts by mapping the original indicator values of all the nodes of the tree to the metric values. Furthermore, since there exists a one-to-one correspondence of the merge tree branches to the original domain, the mapping of the tree induces a mapping of the original indicator field to a new function. The practical advantage of this approach is that in the new metric indicator traditional isosurfaces correspond to a potentially highly sophisticated feature definition but can be extracted with any traditional tool and for various metric values. Note that the metric indicator does not need to be smooth or even continuous as long as it is monotone. For example, evaluating an arbitrary metric and then inflating or deflating its values to enforce monotonicity is a perfectly valid approach.

Relevance. To demonstrate the effectiveness of the general approach we show how relevance - a metric initially introduced to study extinction regions in a turbulent flame [17] — can be described in this framework and used to improve 
vortex detection. Relevance computes a local threshold as the relative difference in the indicator with respect to the local maximum. More formally, for every node $n$ in the tree its relevance $R(n)$ with respect to an indicator $I$ is defined using the relative difference in indicator between a node and the local maximum in its subtree

$$
R(n)=1-\frac{\max \{I(x) \mid x \in \operatorname{subtree}(n)\}-I(n)}{\max \{I(x) \mid x \in \operatorname{subtree}(n)\}-\min (I)} .
$$

Intuitively, relevance describes the relative strength of a vortex compared to its neighborhood. It is not smooth as the local maximum changes discretely at saddle points but it is naturally monotone: Assume that $m, n$ are two nodes of the merge tree part of the same subtree with $I(n)<I(m)$. Let $M_{n}$ and $M_{m}$ be the local maxima of $n$ and $m$ respectively. It follows that $I\left(M_{n}\right) \geq I\left(M_{m}\right)$ which leads to $R(n) \leq R(m)$ :

$$
\begin{aligned}
1-R(m)=\frac{I\left(M_{m}\right)-I(m)}{I\left(M_{m}\right)-\min (I)} & \leq \frac{I\left(M_{m}\right)-I(n)}{I\left(M_{m}\right)-\min (I)} \\
& \leq \frac{I\left(M_{n}\right)-I(n)}{I\left(M_{n}\right)-\min (I)} \\
& \leq \frac{I\left(M_{n}\right)-I(n)}{I\left(M_{n}\right)-\min (I)}=1-R(n) .
\end{aligned}
$$

As will be demonstrated below, this identifies a significantly larger number of well-formed and well-separated vortices than traditional techniques as vortices of all strengths are identified using the same relevance. In practice, this set would likely be filtered by volume, strength or other secondary criteria. While relevance has proven highly useful [17] and simple to compute, other metrics could be substituted. For example, one could extract vortices of equal volume or compute other integrated properties to define local thresholds.

Implementation. A reference implementation of the local thresholds including the relevance transform is publicly available as part of the adaptive thresholds for feature extraction (ADAPT) package at http://github.com/scalability-llnl/ADAPT. The framework uses a variant of [3] which first sorts all vertices according to their indicator function followed by global union-find to construct the tree. One advantage of this scheme is that it produces a fully augmented tree, meaning one that contains not just critical points but all vertices. While this is slightly less efficient (especially in terms of memory) it allows one to compute the chosen metric, (i.e., relevance) for all vertices in the original field. For convenience ADAPT directly outputs the transformed field of the metric indicator which can then be analyzed and processed with any of the standard visualization or analysis tools without modifications. 


\section{Scalar fields for identifying vortices}

For convenience and precision, we turn now to a brief specification of the indicator functions that we extract from the dataset and use to explore the segmentation technique in the next section. These arise from a decomposition of the velocity gradient tensor $\nabla \vec{u}=S+\boldsymbol{\Omega}$ into a symmetric part (rate-of-strain tensor),

$$
S=\frac{1}{2}\left[\nabla \vec{u}+(\nabla \vec{u})^{T}\right],
$$

and an antisymmetric one (vorticity tensor),

$$
\boldsymbol{\Omega}=\frac{1}{2}\left[\nabla \vec{u}-(\nabla \vec{u})^{T}\right] .
$$

The "invariants" of $\nabla \vec{u}[4]$ are

$$
P=u_{i i}, \quad Q=\frac{1}{2}\left(u_{i i}^{2}-u_{i j} u_{j i}\right)=-\frac{1}{2} u_{i j} u_{j i}, \quad R=\operatorname{det}\left(u_{i j}\right) .
$$

Several indicator functions have been proposed; Chakraborty et al. [4] explored the connections between several of these and showed that they are closely related. In the following sections, we will consider several of these commonly referred to as: the $\mathrm{Q}$ criterion, the $\lambda_{2}$ criterion, the $\Delta$ criterion, and the vorticity magnitude. We leave aside others such as the kinematic vorticity number, and the $\mathbf{M}_{Z}$ criterion, proposed by Haller [13]. The $\mathbf{M}_{Z}$ criterion defines a vortex based on Lagrangian considerations; while interesting, here we are focused on Eulerian measures.

The $Q$ criterion. The second invariant, indicating the rotational character, is often used as an indicator of vorticity alone. In compressible flows it is also appropriate to include a pressure criterion, defining a vortex as a region where $Q>0$ and pressure is less than ambient pressure. As discussed by Haller [13], in two dimensions this reduces to an elliptic version of the "Okubo-Weiss" criterion.

The $\lambda_{2}$ criterion. Proposed by Jeong and Hussain [16], the $\lambda_{2}$ criterion defines a vortex core as a connection region with two negative eigenvalues of the symmetric tensor $\left(\boldsymbol{S}^{2}+\boldsymbol{\Omega}^{2}\right)$. This is equivalent to saying a vortex exists in regions where $\lambda_{2}<0$, and $\lambda_{2}$ is the intermediate eigenvalue of $\left(\boldsymbol{S}^{2}+\boldsymbol{\Omega}^{2}\right)$. Although this appears unambiguous, in practice the field is noisy and an alternate threshold is often used. In proposing the $\lambda_{2}$ criterion, the inadequacy of earlier definitions was discussed. Some examples are that the that the $|\omega|$-definition is inappropriate for wall layer flows, that the $|\omega|$-definition can not simultaneously capture rib vortices following an elliptic vortex ring and the main structure due to the large variation in vorticity, and that the $\Delta$-definition (see below) is unable to properly identify a conically symmetric vortex. Jeong et al. also considered various DNS data sets (e.g., of a temporal mixing layer and of a circular jet) to show the improvements made by the $\lambda_{2}$ criterion. 
The $\Delta$ criterion. The $\Delta$ criterion, proposed by Chong et al. [6], defines a vortex as the region of space where the vorticity is sufficiently strong to cause the rate-ofstrain tensor to be dominated by the rotation tensor, i.e., the rate-of-deformation tensor has complex eigenvalues. This occurs when the discriminant is positive:

$$
\Delta=\left(\frac{1}{3} Q\right)^{3}+\left(\frac{1}{2} R\right)^{2}>0 .
$$

Vorticity. The vorticity tensor of the velocity field in our DNS is given by

$$
\omega_{i j}=\frac{1}{2}\left(\frac{\partial u_{i}}{\partial x_{j}}-\frac{\partial u_{j}}{\partial x_{i}}\right),
$$

and the enstrophy by

$$
\Omega=2 \omega_{i j} \omega_{i j} .
$$

The topology for the enstrophy is matched by the vorticity magnitude which we operate on in the following section where the vorticity magnitude is defined as $|\omega|=\left(\frac{1}{2} \Omega\right)^{0.5}$.

\section{Results}

This section will first introduce the test DNS dataset in more detail before comparing vortices identified through relevance with the traditional global thresholds for different indicator functions.

4.1. Test dataset: reacting turbulent jet in crossflow. The test dataset we have chosen is a turbulent reacting jet-in-crossflow configuration described in detail by Grout et al. [11] and depicted in Figure 2. The dataset was constructed using a fully resolved direct numerical simulation (DNS) of a turbulent boundary layer of air with a nitrogen-diluted hydrogen jet introduced into the domain through a nozzle hole on the wall. The domain size was $25 \times 20 \times 20 \mathrm{~mm}$ and a Cartesian grid with $1408 \times 1080 \times 1100$ points was used. The simulation was conducted using S3D [5], a finite-difference code which solves the compressible reacting Navier-Stokes equations with multispecies transport and chemical reaction. The turbulent boundary layer inflow conditions for the three spatial components of the velocity vector were developed by an auxiliary simulation of a periodic flow above a flat plate driven by a pressure gradient. This solution was sampled on a fixed wall-normal planar section of the cross flow that was then used to specify the inflow velocity for the main simulation.

Of note, in addition to the turbulent boundary layer structures, the flow features the jet-in-cross-flow vortical structures identified by Fric [8]: the horseshoe vortices upstream of the jet, the wake vortices between the wall and plume downstream of the jet, the jet shear-layer vortices formed between the jet and the crossflow and the 


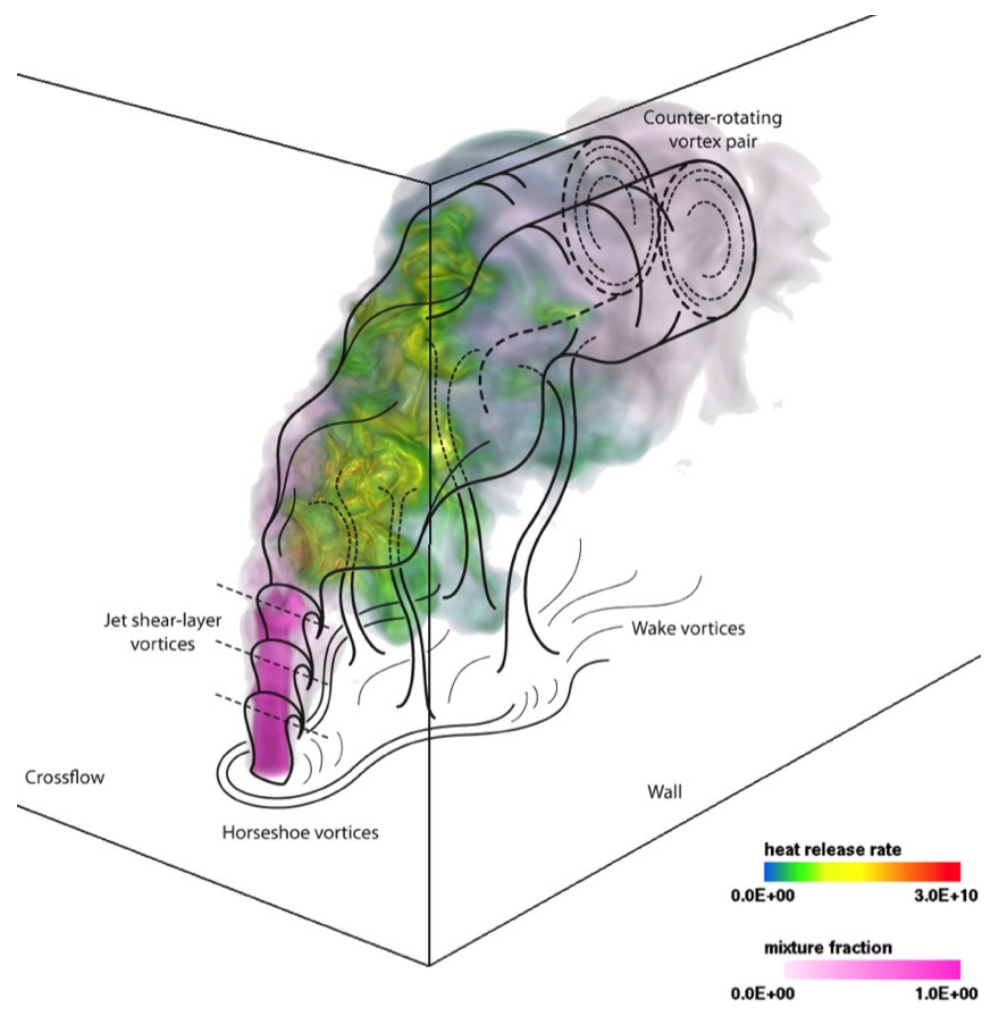

Figure 2. Jet in cross flow test DNS data set [11].

counter-rotating vortex pair in the far-field. In addition, the intense turbulence and vortical structure present in the jet break-down region is interacting with the heatrelease driven expansion (dilatation) from the presence of a flame anchored in the jet [11]. Partially due to the wide dynamic range and multitude vortices embedded in vortices (as in practical turbulent flows), this dataset has resisted analysis using common indicator functions. The approach proposed herein is a formalization of the analysis presented by Grout et al. [10] where topological segmentation of indicator function scalar fields was used to identify vortices for qualitative comparison to the flame position.

4.2. Comparison to previous approaches. Here we compare vortices defined using relevance to determine local thresholds with vortices defined through different global thresholds. It is important to point out that, at their core, merge trees simply allow a convenient abstraction to choose local thresholds. Ultimately, they still depend on the chosen indicator function and inherit all its advantages or disadvantages. Obviously, for any vortex that can be defined using a local threshold, there exists the corresponding global threshold and vice versa. However, what merge trees provide 


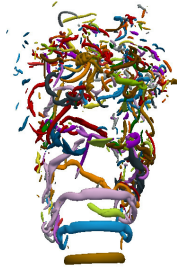

$Q=1000$

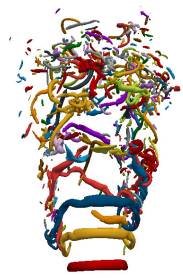

$\lambda_{2}=-1000$

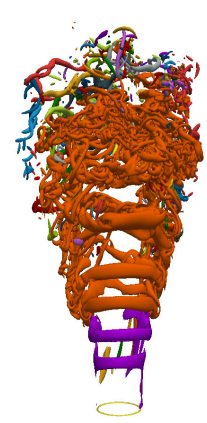

$Q=100$
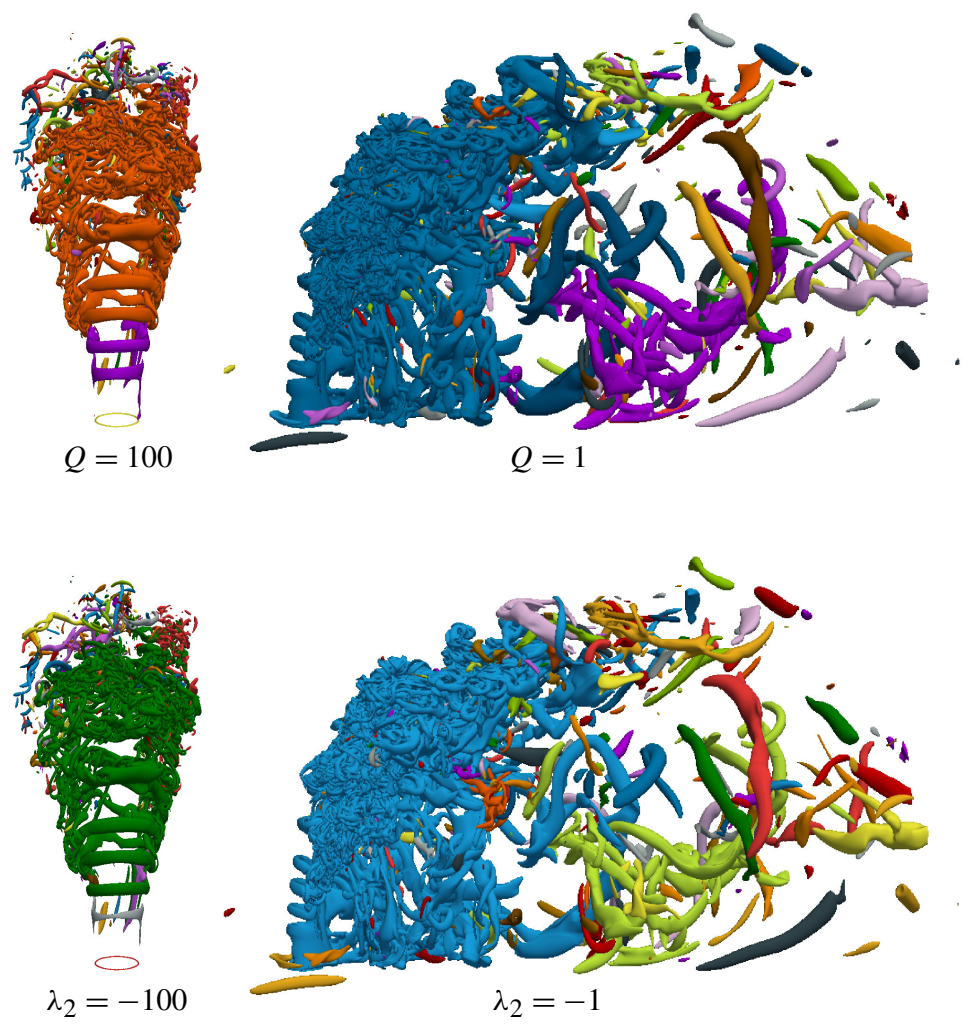

Figure 3. Vortices extracted using $Q$ and $\lambda_{2}$ indicator functions at different thresholds. Vortices are randomly assigned one of 27 colors.

is a means to simultaneously extract all vortices of interest rather than having to explore a wide range of global thresholds. Here we use relevance as a particularly simple metric, but, as discussed above, other approaches could be substituted.

Figures 3 and 4 show vortices extracted using different indicators at various global thresholds. While some are clearly better than others at finding well formed vortices all indicators exhibit the same general behavior. In order to correctly identify the strong shear-layer vortices and those in the center of the flame, very high thresholds are required. However, these exclude all weaker vortices of interest. Yet, lowering the threshold results in the entire breakdown region being erroneously identified as a single vortex. Instead, relevance enables one to extract vortices of all strengths simultaneously as shown in Figure 5. The separation of identification of a vortex existence and strength from any enlargement due to strength is possible because the relevance field encodes local information. At any arbitrary location in the global relevance field, the value indicates the vortex strength relative to the associated local extrema. This theoretically limits the highest value of relevance 


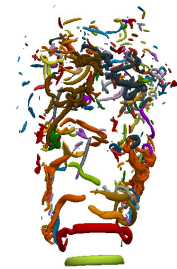

$\Delta=10^{8}$

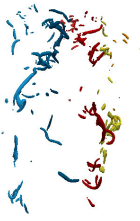

Vort $=5 \times 10^{6}$

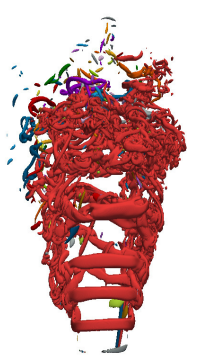

$\Delta=10^{6}$
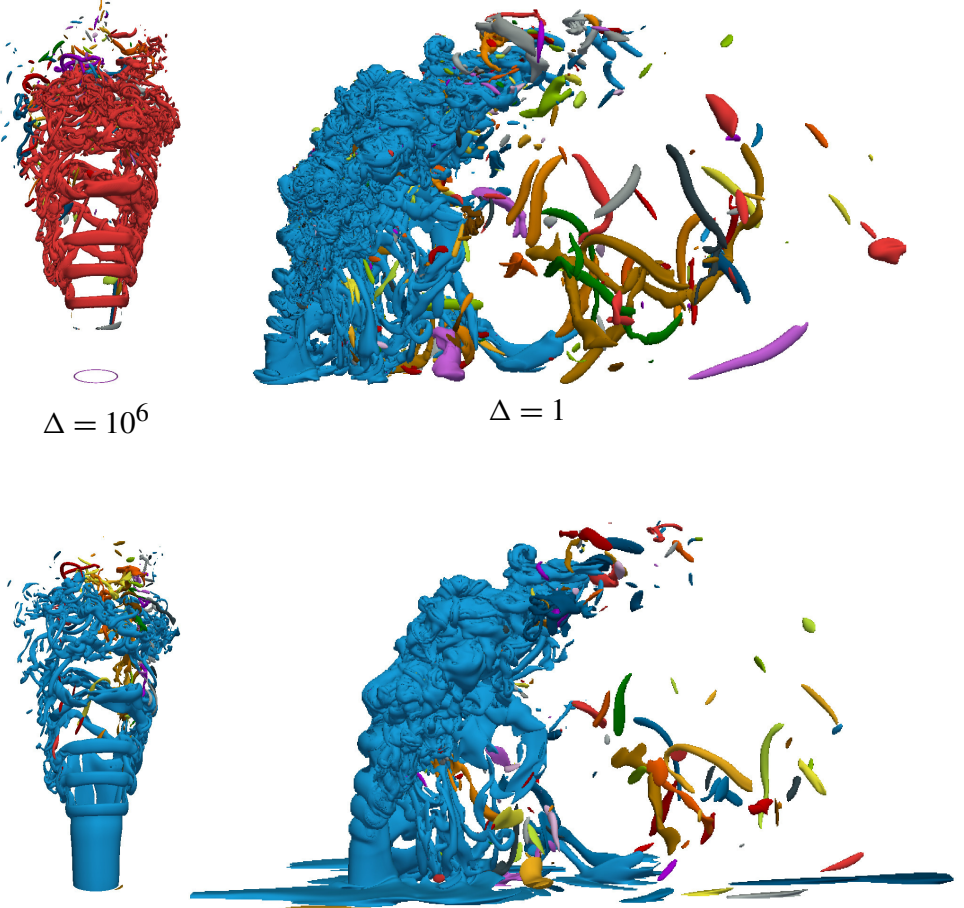

Vort $=2 \times 10^{6}$

Vort $=1 \times 10^{5}$

Figure 4. Vortices extracted using the $\Delta$ and Vorticity indicator functions at different thresholds. Vortices are randomly assigned one of 27 colors.

(largest vortex extent) that can be selected without ambiguity about which vortex is identified, but in practice we have found that all of the indicator functions drop to values far from the local maxima/minima between vortices so this does not seem to pose a problem. This is supported quantitatively by Figure 6, where the number of vortices identified is reasonably insensitive to the relevance threshold over a wide range. Figure 6 also shows the number of vortices each indicator identifies for a range of thresholds for the raw indicator function (i.e., not processed by our algorithm). For the global indicator function thresholds, the number of vortices identified varies significantly and nonmonotonically: slight changes in the threshold cause unpredictable changes in which vortices are considered distinct.

The relevance plots all show the expected behavior of monotonically decreasing vortex counts as a zero relevance identifies all possible seed vortices and lower thresholds progressively merge seeds into more realistic structures. This merging of seeds at low relevance also serves to eliminate numerical noise. The absolute thresholds on the other hand all show significantly fewer individual vortices for most 

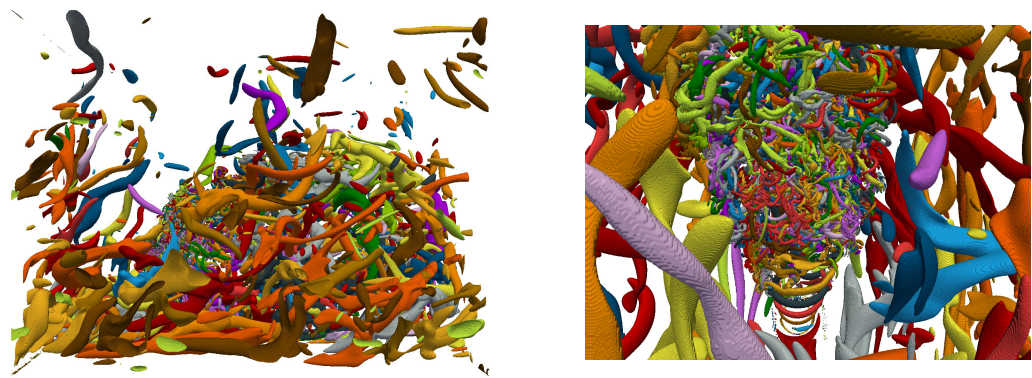

Q criterion at relevance 0.8
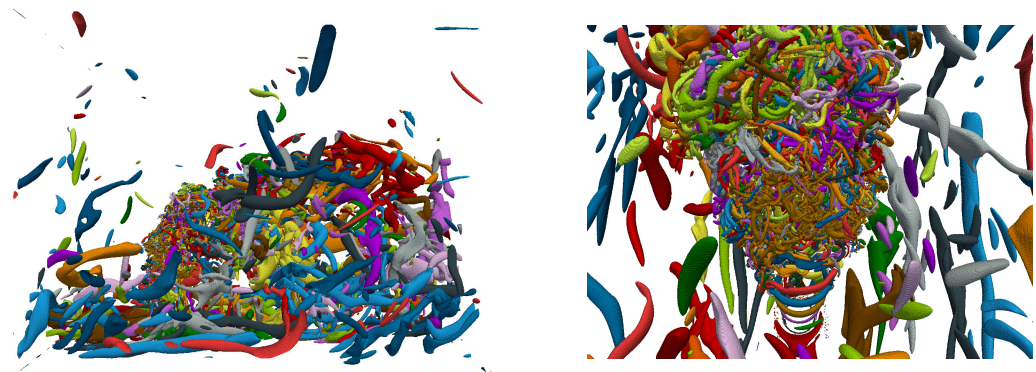

$\lambda_{2}$ criterion at relevance 0.8
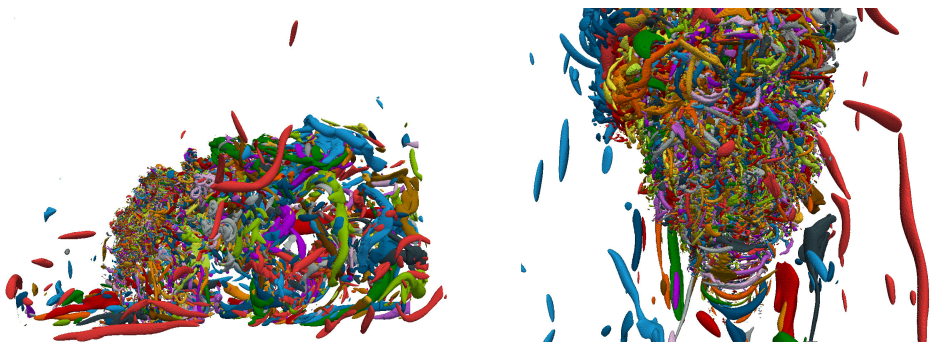

$\Delta$ criterion at relevance 0.8
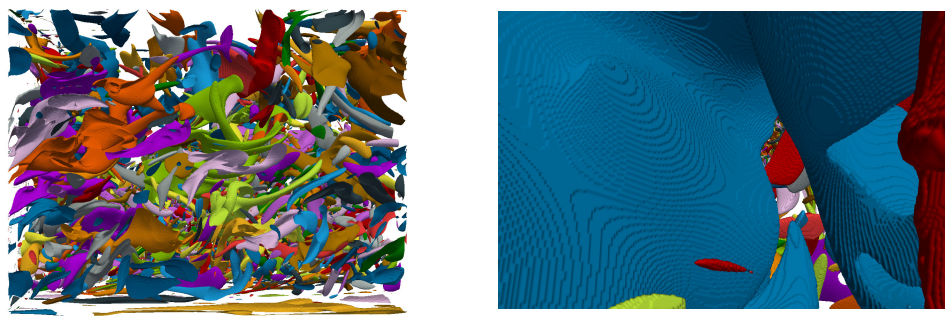

Vorticity criterion at relevance 0.8

Figure 5. Side (left) and zoomed-in front view (right) of the vortices extracted at relevance 0.8 for the $\mathrm{Q}, \lambda_{2}, \Delta$ and Vorticity indicator indicator functions. Both the shear-layer and central flame vortices as well as the low strength vortices on the side and in the back are well separated. $\Delta$ produces similar results to $\mathrm{Q}$ and $\lambda_{2}$ but the vorticity vortices tend to be distorted and pancake-like rather tube-shaped. 

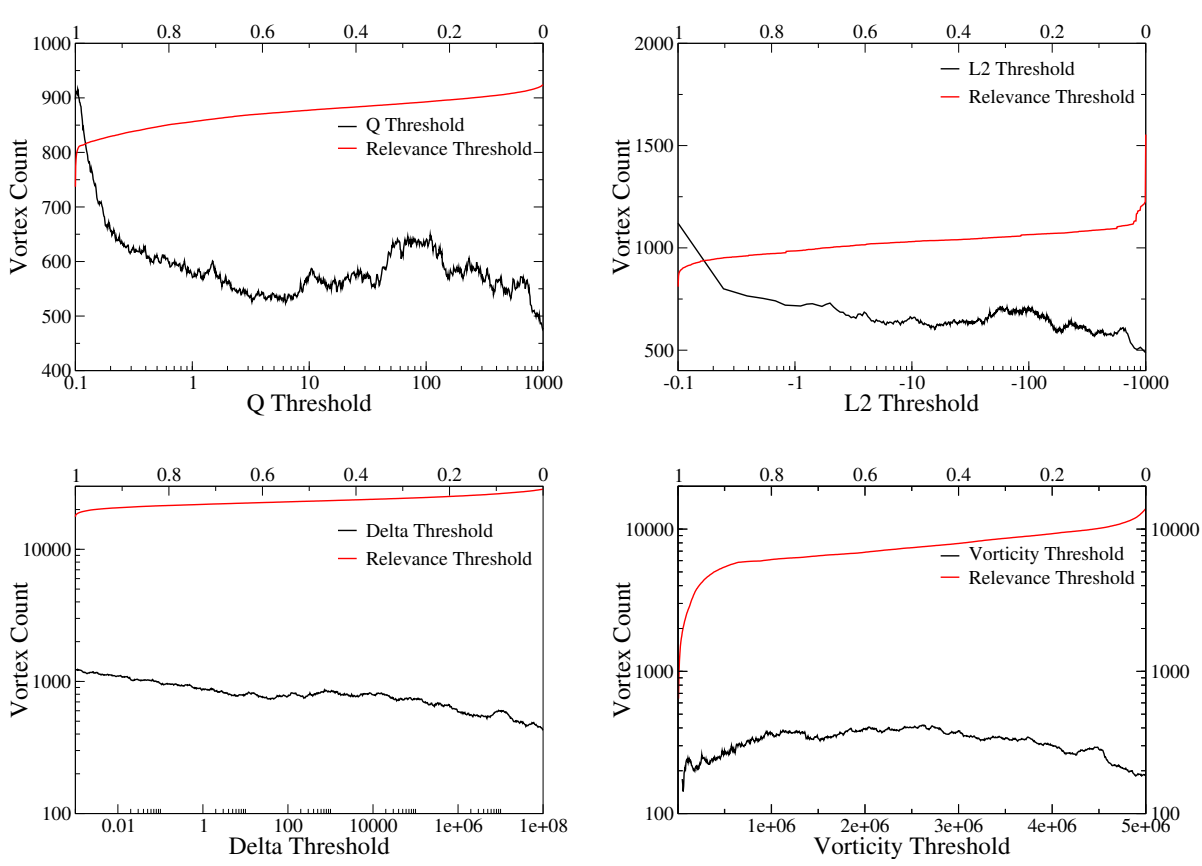

Figure 6. Sensitivity of number of vortices identified to a single scalar threshold and the relevance based threshold. In all cases the number of vortices detected varies smoothly with the relevance parameter (red line, top axis) and is much less sensitive than to the raw threshold (black line, bottom axis).

parameter ranges. Since for lower parameter values significantly more regions are considered vortex-like, this means that simultaneously strong vortices are combined into large superstructures as expected by the images of Figures 3 and 4. To further quantify the differences Figure 7 shows unnormalized cumulative distribution functions (CDFs) of the vortex sizes at different thresholds for vortices covering more than ten voxels to remove artifacts. For all but the $\lambda_{2}$ criterion the relevance thresholds identify significantly more individual vortices and far fewer large outliers. Overall, this confirms the impression of the visualizations that relevance is able to simultaneously detect a much large number of well formed vortices. It is worth noting that while we see separation of identification of the vortices from the process of filtering based on vortex intensity as a positive, information about the strength of the vortex is in some sense lost in this process. It falls to the analyst to use the input fields to label the vortices based on some opportune criteria to indicate their strength. For example, we have found it convenient to first identify vortices based on a relevance criteria and then select vortices based on the strength of the local maxima contained within the each structure. Figure 8 shows the unnormalized $\mathrm{CDF}$ of the per-vortex maximal vorticity for all indicators at the default Relevance 

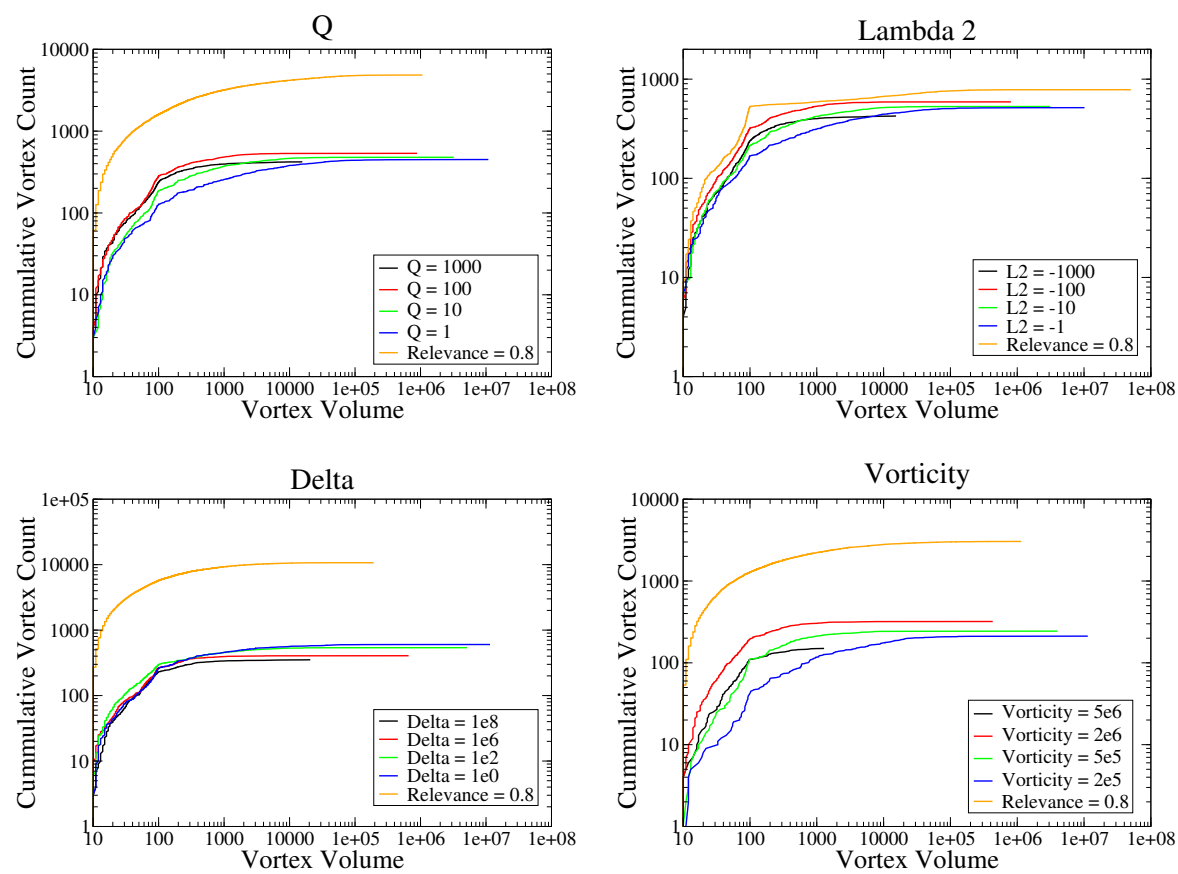

Figure 7. Vortex size distribution for $R=0.8$ compared to distribution for various threshold values for four common indicator functions. In all cases, the relevance metric detects more vortices of each size.

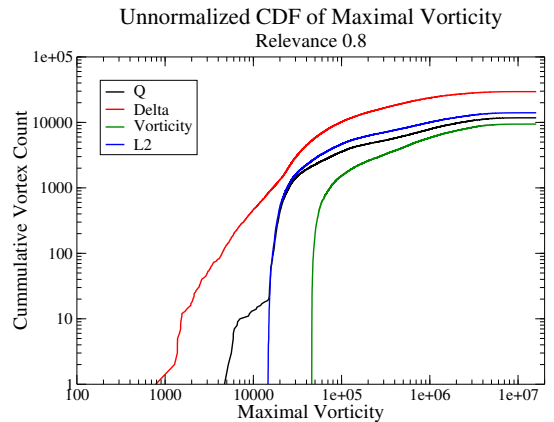

(a)

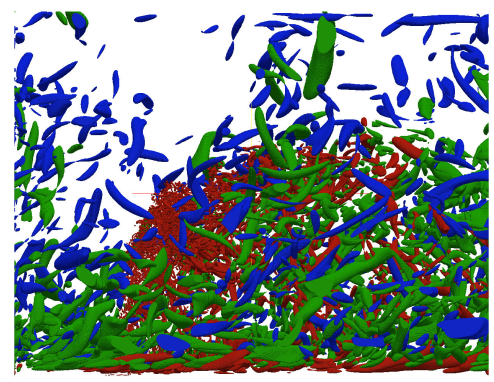

(b)

Figure 8. (a) Unnormalized CDF of maximal per-vortex vorticity for different indicators for $R=0.8$. (b) Rendering of the vortices identified by the $\mathrm{Q}$ criterion color by max vorticity: (red) $\left[10^{5}\right.$, max]; (green) $\left[3 \times 10^{4}, 10^{5}\right]$; and (blue) $\left[0,3 \times 10^{4}\right]$.

of 0.8. All indicators show roughly the same behavior even though the $\Delta$ and Q criteria are able to extract a few noticeably weaker vortices. More important than this specific example is the fact that using local thresholds other selection criteria can be specified individually and without the problem of creating massive artificial 
structures in high indicator regions. Clearly, other criteria such as vortex radius, length, etc, could also be used for subselection. A convenient aspect of encoding the results of the topological analysis in a relevance field is that the single field can then be fed into existing downstream analysis and visualization tools for such analysis.

\section{Conclusion}

While a single isosurface of many traditional measures of vorticity typically combines features (because the strength of the vortices varies rather than because the features are part of the same structure), the proposed approach keeps these separate. By bounding the features by a fraction of the local maximum, we decouple the identification of the vortex extents from the strength of the vortex. Furthermore, because the identification of the vortex extents is insensitive to the value chosen, this ultimately reduces the subjective nature of the analysis. Our approach can be used with any of the standard indicator functions and preserves all their desirable features, such as Galilean invariance. However, the proposed method does not identify "tiered" structures where a large scale rotational motion may contain smaller vortices where the vorticity has valleys inside of the largest structure. This is a direct consequence of basing the segmentation on scalar fields designed to extract regions of consistently dominant rotational motion. When Jeong and Hussain [16] proposed the $\lambda_{2}$ criterion, two of the primary criticisms of earlier methods were the treatment of the conical vertex and the inability to simultaneously represent vortices with a large variation in strength. Our method, when applied to the $\lambda_{2}$ fields inherits improvements made in both of these areas. As the $\lambda_{2}$ field in practice tends to be very noisy, it is still limited in the second respect. However, when segmented through a topological approach, it is easy to "clean up" for unambiguous identification of structures. In addition, the topological approach also addresses the difficulty of using the vorticity magnitude to simultaneously represent vortices with widely varying strengths.

\section{Acknowledgements}

Sandia National Laboratories is a multiprogram laboratory managed and operated by Sandia Corporation, a wholly owned subsidiary of Lockheed Martin Corporation, for the U.S. Department of Energy's National Nuclear Security Administration under contract DE-AC04-94AL85000. This research used resources of the Oak Ridge Leadership Computing Facility at the Oak Ridge National Laboratory, which is supported by the Office of Science of the U.S. Department of Energy under contract no. DE-AC05-00OR22725. This material is based upon work supported by the U.S. Department of Energy's Office of Science and Office of Advanced 
Scientific Computing Research. This work was supported by the U.S. Department of Energy under Contract No. DE-AC36-08-GO28308 with the National Renewable Energy Laboratory.

\section{References}

[1] W. T. Ashurst, A. R. Kerstein, R. M. Kerr, and C. H. Gibson, Alignment of vorticity and scalar gradient with strain rate in simulated Navier-Stokes turbulence, Phys. Fluids 30 (1987), no. 8, 2343-.

[2] J. Bennett, V. Krishnamurthy, S. Liu, V. Pascucci, R. Grout, J. Chen, and P.-T. Bremer, Featurebased statistical analysis of combustion simulation data, IEEE Trans. Vis. Comp. Graph. 17 (2011), no. 12, 1822-1831.

[3] H. Carr, J. Snoeyink, and U. Axen, Computing contour trees in all dimensions, Comput. Geom. 24 (2003), no. 2, 75-94. MR 2004b:68162 Zbl 1052.68098

[4] P. Chakraborty, S. Balachandar, and R. J. Adrian, On the relationships between local vortex identification schemes, J. Fluid Mech. 535 (2005), 189-214. MR 2007f:76118

[5] J. H. Chen, A. Choudhary, B. de Supinski, M. DeVries, E. R. Hawkes, S. Klasky, W.K.Liao, K. L. Ma, J. Mellor-Crummey, N. Podhorszki, R. Sankaran, S. Shende, and C. S. Yoo, Terascale direct numerical simulations of turbulent combustion using S3D, Comput. Sci. Disc. 2 (2009), 015001.

[6] M. S. Chong, A. E. Perry, and B. J. Cantwell, A general classification of three-dimensional flow fields, Phys. Fluids A 2 (1990), no. 5, 765-777. MR 92b:76037

[7] J. del Álamo, J. Jimenez, P. Zandonade, and R. D. Moser, Self-similar vortex clusters in the turbulent logarithmic region, J. Fluid Mech. 561 (2006), 329-358. Zbl 1157.76346

[8] T. F. Fric and A. Roshko, Vortical structure in the wake of a transverse jet, J. Fluid Mech. 279 (1994), 1-47. Zbl 1303.11056

[9] C. H. Gibson, Isoenstrophy points and surfaces in turbulent flow and mixing, Fluid Dynamics Res. 3 (1988), no. 1-4, 331-336.

[10] R. W. Grout, P.-T. Bremer, A. Gruber, J. Bennett, A. Gyulassy, and J. H. Chen, Heat release and turbulence statistics from a DNS of reacting jet in cross-flow parameterized in a jet natural coordinate system developed from scalar quantities, paper at international conference on numerical combustion, corfu, 2011; sandia national laboratories report no. SAND2011-2994C/482316, Sandia National Laboratories, Livermore, CA, 2011.

[11] R. Grout, A. Gruber, H. Kolla, P.-T. Bremer, J. Bennett, A. Gyulassy, and J. Chen, A direct numerical simulation study of turbulence and flame structure in transverse jets analysed in jet-trajectory based coordinates, J. Fluid Mech. 706 (2012), 351-383. Zbl 1275.76147

[12] R. Grout, A. Gruber, C. Yoo, and J. Chen, Direct numerical simulation of flame stabilization downstream of a transverse fuel jet in cross-flow, Proc. Combustion Inst. 33 (2011), no. 1, 1629-1637.

[13] G. Haller, An objective definition of a vortex, J. Fluid Mech. 525 (2005), 1-26. MR 2008h:76007 Zbl 1071.76015

[14] J. C. R. Hunt, A. Wray, and P. Moin, Eddies, stream, and convergence zones in turbulent flows, Proceedings of the summer program 1988, Center for Turbulence Research (Stanford University), 1988, pp. 193-208.

[15] A. Hussain, Coherent structures - reality and myth, Phys. Fluids 26 (1983), 2816-2850. 
[16] J. Jeong and F. Hussain, On the identification of a vortex, J. Fluid Mech. 285 (1995), 69-94. MR 96a:76017 Zbl 0847.76007

[17] A. Mascarenhas, R. W. Grout, C. S. Yoo, and J. H. Chen, Tracking flame base movement and interaction with ignition kernels using topological methods, J. Phys. Conf. Ser. 180 (2009), 012086.

[18] M. Tanahashi, T. Miyauchi, and J. Ikeda, Scaling law of coherent fine scale structure in homogeneous isotropic turbulence, Proceedings of the 11th Symposium on Turbulent Shear Flows, I, Institut National Polytechnique, Centre National de la Recherche Scientifique, Université Joseph Fourier, 1997, pp. 4-17.

[19] H. Tennekes and J. Lumley, A first course in turbulence, MIT Press, Cambridge, MA, 1972. Zbl 0285.76018

Received October 2, 2014. Revised September 17, 2015.

PEER-TIMO BREMER: bremer5@llnl.gov

Center for Applied Scientific Computing, Lawrence Livermore National Laboratories,

7000 East Avenue, L-422, Livermore, CA 94551, United States

ANDREA GRUBER: Andrea.Gruber@sintef .no

SINTEF Energy Research, K. Heiesvei 1A, 7465 Trondheim, Norway

JANINE C. BENNETT: jcbenne@sandia.gov

Sandia National Laboratories, 7011 East Ave, Livermore, CA 94551, United States

ATTILA GyUlaSSY: aggyulassy@ucdavis .edu

University of Utah, Salt Lake City, CA 84112, United States

Hemanth Kolla: hnkolla@sandia.gov

Sandia National Laboratories, 7011 East Ave, Livermore, CA 94551, United States

JACQUELINE H. CHEN: jhchen@sandia.gov

Reacting Flows, Sandia National Laboratories, 7011 East Ave, Livermore, CA 94551, United States

RAY W. GROUT: ray.grout@nrel.gov

Computational Science Center, National Renewable Energy Laboratory, 15013 Denver West Parkway,

Golden, CO 80401, United States 


\title{
Communications in Applied Mathematics and Computational Science
}

\author{
msp.org/camcos
}

EDITORS

MANAGING EDITOR

John B. Bell

Lawrence Berkeley National Laboratory, USA

jbbell@lbl.gov

\section{BOARD OF EDITORS}

\begin{tabular}{|c|c|c|c|}
\hline Marsha Berger & $\begin{array}{l}\text { New York University } \\
\text { berger@cs.nyu.edu }\end{array}$ & Ahmed Ghoniem & $\begin{array}{l}\text { Massachusetts Inst. of Technology, USA } \\
\text { ghoniem@mit.edu }\end{array}$ \\
\hline Alexandre Chorin & $\begin{array}{l}\text { University of California, Berkeley, USA } \\
\text { chorin@math.berkeley.edu }\end{array}$ & Raz Kupferman & $\begin{array}{l}\text { The Hebrew University, Israel } \\
\text { raz@math.huji.ac.il }\end{array}$ \\
\hline Phil Colella & $\begin{array}{l}\text { Lawrence Berkeley Nat. Lab., USA } \\
\text { pcolella@lbl.gov }\end{array}$ & Randall J. LeVeque & $\begin{array}{l}\text { University of Washington, USA } \\
\text { rj1@amath.washington.edu }\end{array}$ \\
\hline Peter Constantin & $\begin{array}{l}\text { University of Chicago, USA } \\
\text { const@cs.uchicago.edu }\end{array}$ & Mitchell Luskin & $\begin{array}{l}\text { University of Minnesota, USA } \\
\text { luskin@umn.edu }\end{array}$ \\
\hline Maksymilian Dryja & $\begin{array}{l}\text { Warsaw University, Poland } \\
\text { maksymilian.dryja@acn.waw.pl }\end{array}$ & Yvon Maday & $\begin{array}{l}\text { Université Pierre et Marie Curie, France } \\
\text { maday@ann.jussieu.fr }\end{array}$ \\
\hline M. Gregory Forest & $\begin{array}{l}\text { University of North Carolina, USA } \\
\text { forest@amath.unc.edu }\end{array}$ & James Sethian & $\begin{array}{l}\text { University of California, Berkeley, USA } \\
\text { sethian@ math.berkeley.edu }\end{array}$ \\
\hline Leslie Greengard & $\begin{array}{l}\text { New York University, USA } \\
\text { greengard@cims.nyu.edu }\end{array}$ & Juan Luis Vázquez & $\begin{array}{l}\text { Universidad Autónoma de Madrid, Spain } \\
\text { juanluis.vazquez@uam.es }\end{array}$ \\
\hline Rupert Klein & $\begin{array}{l}\text { Freie Universität Berlin, Germany } \\
\text { rupert.klein@pik-potsdam.de }\end{array}$ & Alfio Quarteroni & $\begin{array}{l}\text { Ecole Polytech. Féd. Lausanne, Switzerland } \\
\text { alfio.quarteroni@epfl.ch }\end{array}$ \\
\hline \multirow[t]{2}{*}{ Nigel Goldenfeld } & $\begin{array}{l}\text { University of Illinois, USA } \\
\text { nigel@uiuc.edu }\end{array}$ & Eitan Tadmor & $\begin{array}{l}\text { University of Maryland, USA } \\
\text { etadmor@cscamm.umd.edu }\end{array}$ \\
\hline & & Denis Talay & $\begin{array}{l}\text { INRIA, France } \\
\text { denis.talay@inria.fr }\end{array}$ \\
\hline
\end{tabular}

\section{PRODUCTION}

production@msp.org

Silvio Levy, Scientific Editor

See inside back cover or msp.org/camcos for submission instructions.

The subscription price for 2016 is US $\$ 95 /$ year for the electronic version, and $\$ 135 /$ year $(+\$ 15$, if shipping outside the US) for print and electronic. Subscriptions, requests for back issues from the last three years and changes of subscribers address should be sent to MSP.

Communications in Applied Mathematics and Computational Science (ISSN 2157-5452 electronic, 1559-3940 printed) at Mathematical Sciences Publishers, 798 Evans Hall \#3840, c/o University of California, Berkeley, CA 94720-3840, is published continuously online. Periodical rate postage paid at Berkeley, CA 94704, and additional mailing offices.

CAMCoS peer review and production are managed by EditFLOW ${ }^{\circledR}$ from MSP.

\section{PUBLISHED BY}

mathematical sciences publishers

nonprofit scientific publishing

http://msp.org/

(C) 2016 Mathematical Sciences Publishers 


\section{Communications in Applied Mathematics and Computational Science}

A front-tracking shock-capturing method for two gases

Mehdi Vahab and Gregory H. Miller

Identifying turbulent structures through topological segmentation

Peer-Timo Bremer, Andrea Gruber, Janine C. Bennett,

Attila Gyulassy, Hemanth Kolla, Jacqueline H. Chen and

RAY W. GROUT

An asymptotic-preserving scheme for systems of conservation laws with source terms on 2D unstructured meshes

Christophe Berthon, Guy Moebs, Céline Sarazin-Desbois and Rodolphe Turpault

An immersed boundary method for rigid bodies

Bakytzhan Kallemov, Amneet Pal Singh Bhalla, Boyce E.

Griffith and Aleksandar DoneV 